\title{
Análisis de la innovación en las cooperativas mediante la captación del valor intangible: un estudio empírico
}

\author{
Pedro Carmona, Julián Martínez y José Pozuelo
}

\section{RESUMEN}

Las empresas cooperativas al igual que las capitalistas deben apostar por la innovación en productos, servicios y procesos de producción para mantener e incrementar su posición competitiva, máxime si se tiene en cuenta que para ello se considera fundamental la existencia de un capital humano capaz de asimilar los cambios derivados de estos procesos. En este sentido, las cooperativas cuentan con una singular herramienta, que es el fondo de educación, formación y promoción, dotado a partir de los excedentes cooperativos y concebido precisamente para que sus empleados se formen adecuadamente, según las exigencias laborales de cada momento, pudiendo adaptarse más rápidamente a los cambios que conlleva la innovación.

En este trabajo pretendemos analizar la influencia de determinados indicadores relacionados con la rentabilidad, la solvencia, el endeudamiento, el crecimiento y el número de empleados en la creación de valor de activos intangibles que evidencian un mayor apuesta por la innovación. Para ello analizamos los datos económico-financieros de una muestra de empresas cooperativas no financieras activas entre 2009 y 2011.

PALABRAS CLAVE: Cooperativas, innovación, activos intangibles, análisis.

CLAVES ECONLIT: P130, M210, 0310.

Cómo citar este artículo: CARMONA, P., MARTÍNEZ, J. \& POZUELO, J. (2015): "Análisis de la innovación en las cooperativas mediante la captación del valor intangible: un estudio empírico", CIRIECEspaña, Revista de Economía Pública, Social y Cooperativa, 84, 163-193.

Correspondencia: Pedro Carmona, Julián Martínez y José Pozuelo, Profesores Titulares de Universidad, Departamento de Contabilidad, Facultad de Economía, Universidad de Valencia. Av. de los Naranjos s/n, 46022 Valencia. Email de contacto: pedro.carmona@uv.es. 


\title{
Analyse de l'innovation dans les coopératives par le biais de la captation de la valeur intangible : une étude empirique
}

RÉSUMÉ : Les entreprises coopératives, tout comme les entreprises capitalistes, doivent miser sur l'innovation en matière de produits, services et processus de production pour maintenir et améliorer leur compétitivité, ce qui est d'autant plus vrai si on considère que l'existence d'un capital humain capable d'assimiler les changements dérivés de ces processus est essentielle pour elles. En ce sens, les coopératives disposent d'un outil unique : le fonds pour l'éducation, la formation et la promotion, financé à partir des excédents des coopératives et conçu précisément pour que leurs employés se forment correctement, selon les exigences professionnelles de chaque instant, et ainsi s'adaptent plus rapidement aux changements résultant de l'innovation.

L'objectif de ce travail est d'analyser l'influence de certains indicateurs en lien avec la rentabilité, la solvabilité, l'endettement, la croissance et le nombre d'employés dans la création de la valeur d'actifs incorporels montrant un meilleur engagement pour l'innovation. Nous analysons donc les données économiques et financières d'un échantillon d'entreprises coopératives non financières actives entre 2009 et 2011.

MOTS CLÉ : Coopératives, innovation, actifs incorporels, analyse.

\section{An analysis of innovation in cooperatives through intangible value}

\begin{abstract}
Cooperatives, like any other enterprise, need to make a commitment to innovation in products, services and production processes in order to maintain and improve their competitive position, taking into account in particular that it is essential for their human capital to be capable of assimilating the changes resulting from these processes. From this point of view, they have an exceptional instrument in the education, training and promotion fund, endowed from the cooperative surpluses precisely with the purpose of training the employees to meet the demands of the work being done at any given time, so they can adapt faster to the changes required by innovation.

The aim of this study was to analyse the influence of certain indicators, related to profitability, solvency, debt, growth and the number of employees, on value creation in intangible assets that show a greater commitment to innovation. In order to identify differences between innovative and non-innovative enterprises in a sample of nonfinancial cooperatives, univariate and multivariate analyses were performed on these financial indicators for the 2009-2011 period.
\end{abstract}

KEY WORDS: Cooperatives, innovation, intangible assets, analysis. 


\section{ANÁLISIS DE LA INNOVACIÓN EN LAS COOPERATIVAS MEDIANTE LA CAPTACIÓN DEL VALOR INTANGIBLE: UN ESTUDIO EMPÍRICO}

\section{1.- Introducción}

La progresiva globalización de los mercados y la lucha por la supervivencia en el entorno de la crisis actual plantea a las empresas la necesidad de adoptar nuevas estrategias dirigidas a conseguir aumentos de competitividad. Ello les permitirá expandirse aprovechado las nuevas oportunidades que ofrecen los mercados emergentes, sobre todo de Asia y de Europa del Este. Para lograrlo es fundamental establecer y asumir procesos continuados de innovación con los que conseguir nuevos productos y servicios, mejor calidad y precios más bajos que garanticen una posición competitiva permanente y viable.

Para converger con los países líderes en innovación, que en algunos casos son los principales competidores en los mercados internacionales, la Comisión Europea en el marco de la Estrategia Europa 2020 ha establecido como objetivo alcanzar un nivel de inversión total (pública y privada) en I + D del 3\% del PIB de la Unión Europea. Sin embargo, en nuestro país, tras una década de crecimiento continuo, el gasto en I + D + i ha disminuido del 1,38\% al 1,30\% del PIB entre 2009 y 2012 (CEOE, 2014) lo que no está acorde con las propuestas de la Unión Europea. Con ello, nos alejamos del discurso por parte de las autoridades gubernamentales y de la opinión pública de considerar el cambio del modelo productivo como requisito fundamental para salir de la crisis económica y conseguir un crecimiento sostenible y equilibrado.

Las empresas cooperativas, que tienen un marcado carácter social y se rigen por una serie de principios o características que las diferencian de las sociedades mercantiles o capitalistas, no deben ser ajenas a estos procesos de innovación y aprovechamiento de las nuevas tecnologías de la información, máxime si se tiene en cuenta que uno de esos principios fundamentales es el de educación y promoción cooperativa. Con él se pretende que los socios y trabajadores logren un alto nivel de especialización que redundará en una producción más eficiente, bien porque ésta sea de mayor calidad o bien por la obtención de un coste laboral más bajo que en otras empresas. El desempeño laboral resulta clave en estas empresas hasta el punto que, según Bretones (2004), la necesidad de empleo subyace en la mayor creación de cooperativas de trabajadores en los períodos de crisis económica.

También, a partir del principio de la participación económica de los socios, éste es consciente que no obtiene más rentas el que más capital ha aportado a la cooperativa sino el que más actividad ha realizado. Ante esta circunstancia el socio debe percibir que no es solamente la cooperativa la que obtiene el beneficio, pues una parte del mismo va a parar a él en función de su trabajo. En consecuencia, se esforzará más a la hora de operar con ella, consiguiendo la cooperativa un volumen óptimo de actividad o por lo menos más que suficiente para poder seguir compitiendo. En este sentido, estas 
empresas cuentan con el fondo de educación, formación y promoción, dotado a partir de los excedentes cooperativos, concebido precisamente para que sus empleados se formen adecuadamente y asimilen correctamente los cambios derivados del proceso innovador.

Partiendo de esta base, en este trabajo realizamos en primer lugar una revisión de la literatura económica sobre aspectos que relacionan el capital intangible con la inversión en I + D + i y su influencia en el crecimiento económico. Paralelamente justificamos la relación directa existente entre el proceso innovador y los activos intangibles de una empresa. A continuación, realizamos un estudio empírico que pretende analizar la influencia de determinados indicadores relacionados con la rentabilidad, solvencia, endeudamiento, crecimiento y el número de empleados en la creación de valor de activos intangibles que, en definitiva, evidencian una mayor apuesta por la innovación. Para ello, partimos de una muestra de empresas cooperativas no financieras activas entre 2009 y 2011 aplicando diferentes técnicas estadísticas, univariantes y multivariantes, que han permitido demostrar la conexión entre los indicadores empleados y las evidencias de innovación a partir de los activos intangibles.

Como detallaremos a lo largo del trabajo, los resultados obtenidos muestran, en general, que las empresas innovadoras tienen un mejor desempeño económico y financiero que las empresas no innovadoras.

\section{2.- Antecedentes}

De acuerdo con Pulido (2009), es a partir de los años 90 del siglo pasado cuando se multiplican los trabajos sobre la relación entre crecimiento económico y diferentes componentes de capital intangible, tales como capital tecnológico o de conocimiento (principalmente medido a través de esfuerzos en I + D o patentes), capital humano (principalmente ponderación de la fuerza de trabajo según el nivel de estudios formales alcanzado) y capital social (medido indirectamente por indicadores sobre estabilidad política, eficiencia administrativa, seguridad ciudadana, etc.). En este sentido, en Barro y Salai-Martín (1995) se presentan varios modelos donde se relacionan ritmos de crecimiento con esfuerzos en avances tecnológicos, capital humano o variables del entorno socio-político. Nelson (1996) señala la I + D y el capital humano como claves del crecimiento económico, estableciendo que si los trabajadores tienen una mayor preparación educativa serán más flexibles y capaces de aprender una variedad de ocupaciones, el ajuste de empleos más antiguos a otros nuevos, de sectores en declive a otros en expansión, rompiendo la resistencia al cambio tecnológico que puede suponer un proceso de innovación. Otros trabajos relacionan la inversión en I + D con el crecimiento en las ventas (Dowling y MacGee, 1994), el éxito financiero (Capon et al., 1990), o consideran que es necesario un concepto 
multidimensional más amplio de estrategia de innovación y su relación con el resultado (Zahra y Covin, 1993; Zahra y Bogner, 1999).

Más tarde, investigadores como Lev (2001) realizan estudios donde consideran el valor de los intangibles como un "proxy" de la actividad innovadora, de la misma forma que autores como Nakamura (2001), Corrado et al. (2006), Hill and Youngman (2003) y Arundel (2007) asimilan los gastos en activos intangibles a inversiones en capacidad innovadora y, aunque no podamos considerar que una inversión en intangibles sea necesariamente una inversión en innovación, sí es cierto que el nuevo conocimiento derivado puede conducir a desarrollar una invención que sólo tendrá valor económico cuando haya sido comercializada produciendo beneficios a la empresa. Guijarro y Moya (2006) analizan qué variables de la información pública de los estados contables de las empresas tecnológicas se relacionan con la inversión en intangibles. Para ello se basan en empresas cotizadas en el índice Nasdaq-100 durante el período 1999-2004, concluyendo que las empresas con mayor factor intangible logran mejores resultados económicos y que las empresas de menor tamaño realizan proporcionalmente un mayor esfuerzo de innovación respecto de las más consolidadas, que estarían dispuestas a arriesgar menos. Rosenbusch et al. (2011) presentan un trabajo donde a partir de los datos aportados por diferentes estudios empíricos realizados en países tan diversos como Estados Unidos, Reino Unido, China, Francia, Irlanda, Turquía o Taiwán establecen una relación positiva entre el rendimiento de las pequeñas y medianas empresas y los procesos de innovación, medidos fundamentalmente por la inversiones en I + D.

En España, los trabajos sobre innovación se han basado por lo general en análisis de datos obtenidos a través de cuestionarios (Guzmán y Martínez, 2008; COTEC, 2010). La mayoría se han ubicado en determinadas zonas geográficas (Fernández y Peña; 2009; Águila y Padilla, 1010; Basterretxea y Martínez, 2012; Rodríguez y Guzmán, 2013; Gómez y Aleixandre, 2014).

Por otra parte, acorde con la línea de investigación basada en los intangibles, debemos hacer referencia al trabajo de Carmona et al. (2012) donde se analiza el proceso innovador de las PYMES españolas del sector servicios (excluyendo empresas financieras) a través de la valoración de los activos intangibles en los estados financieros en los ejercicios 2007-2009. Los resultados obtenidos mediante análisis estadístico muestran que las empresas consideradas innovadoras presentan generalmente unos ratios económico-financieros con valores mejores que las no innovadoras, destacando las diferencias en actividad, presión fiscal y crecimiento a favor en las innovadoras y en solvencia a corto y largo plazo a favor de las no innovadoras. 


\section{3.- El proceso innovador}

En la actualidad, prácticamente hay unanimidad en pensar que el modelo productivo español debe cambiar incorporando una mayor tasa de inversión en $I+D+i$, aunque las condiciones económicas no sean las más favorables. Este déficit de inversión en I + D + i se pone de manifiesto si, tomando como referencia la inversión en intangibles, nos comparamos con otros países de referencia como Estados Unidos y Reino Unido. Lo apreciamos en la Figura 1.

Por ello, las medidas de política fiscal han pretendido poner un mayor énfasis en los incentivos fiscales por la realización de este tipo de actividades. Destacan la recuperación de la deducción por actividades de investigación y desarrollo e innovación tecnológica (RDL 3/2009), el aumento del límite de cuota íntegra y el porcentaje de deducción (Ley 2/2011) y la propuesta de nuevos estímulos en la ley de emprendedores (Ley 14/2013). En definitiva, se reconoce que el esfuerzo en investigación, desarrollo e innovación del sector privado en España es inferior al de los países de nuestro entorno, debiendo incentivarse por considerarse esencial para el crecimiento y la competitividad de un país. En consonancia con este mandato, la nueva Ley del Impuesto sobre Sociedades (Ley 27/2014), mantiene la de investigación, desarrollo e innovación tecnológica a pesar de eliminar la mayoría de las deducciones y, al conservar los porcentajes de deducción de la normativa anterior, la minoración del tipo de gravamen se interpreta como un incremento efectivo de este incentivo.

\section{Figura 1. La inversión en tangibles y en intangibles (\% PIB 2009)}

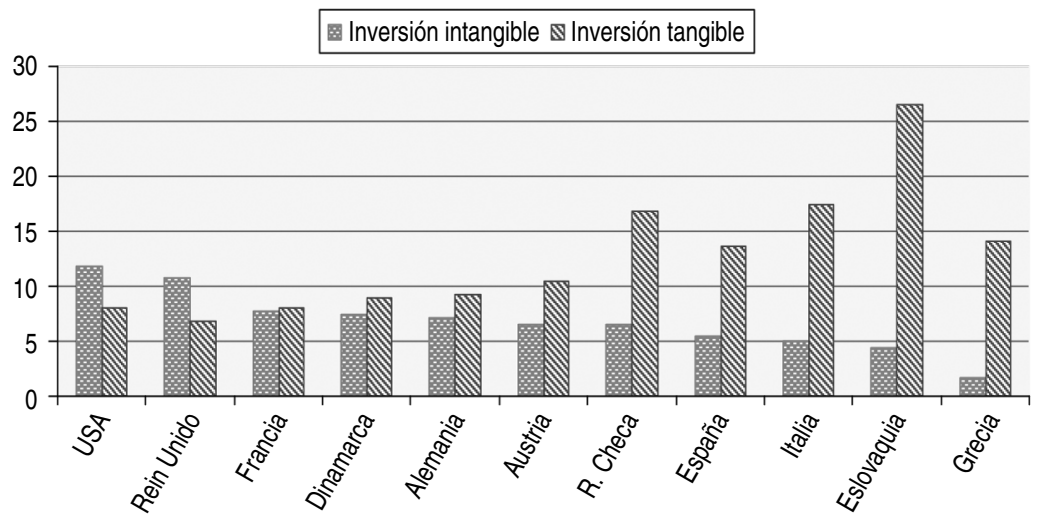

FUENTE: COINVEST Project (www.coinvest.org.uk). 
La innovación ha sido definida como un proceso complejo y creativo, esencial para la economía, que comprende la creación de un nuevo producto, la utilización de un nuevo proceso en la producción o la introducción de un nuevo método de gestión organizativo o comercial (Schumpeter, 1976). En términos similares se manifiesta la OCDE (2007) cuando establece que la innovación consiste en "la introducción de un producto (bien o servicio) o de un proceso, nuevo o significativamente mejorado, o la introducción de un método de comercialización o de organización nuevo, aplicado a las prácticas de negocio, a la organización del trabajo o a las relaciones externas". En definitiva, podemos establecer que innovar significa cambiar con el objetivo de crear valor en la empresa tal como se describe en la Figura 2.

La innovación puede ser tecnológica y no tecnológica y supondrá novedades o cambios de tipo científico, tecnológico, organizacional, comercial o en marketing. Los incrementos de valor generados en la empresa pueden ser de tipo material o intangible, teniendo en cuenta además que el incremento de valor intangible supondrá de forma indirecta una mejora o incremento de valor de los elementos materiales de la empresa.

\section{Figura 2. El proceso innovador}

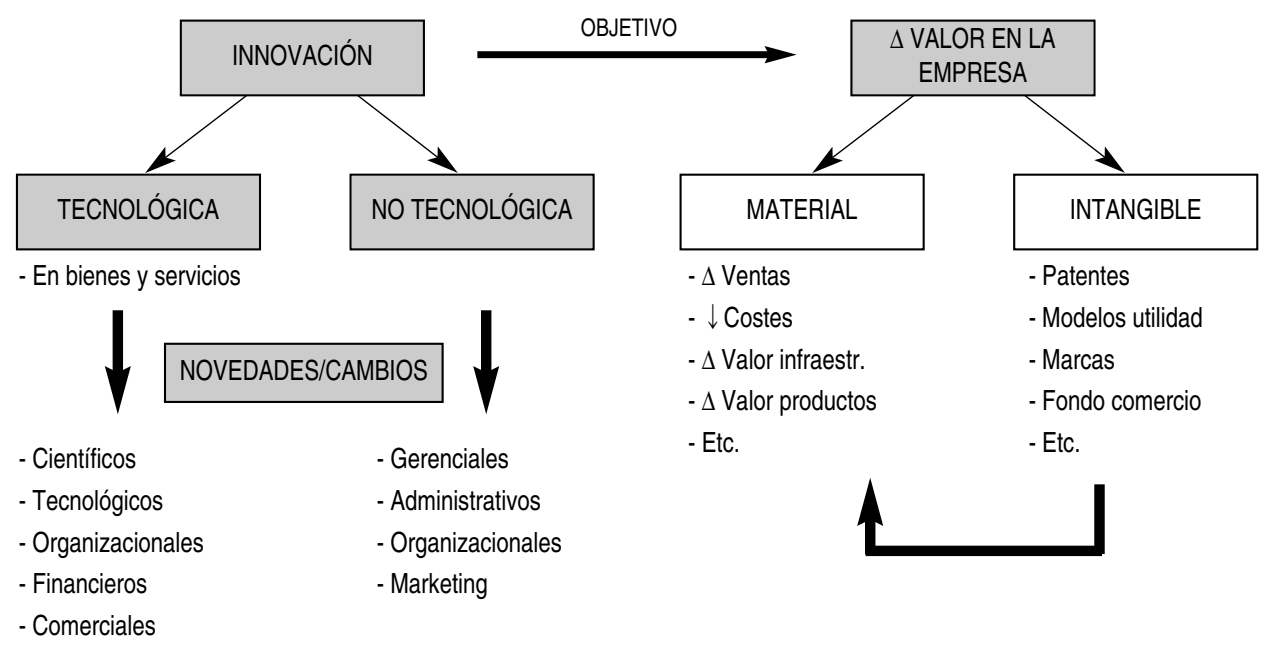

FUENTE: Elaboración propia.

Si bien es cierto que la contabilización de activos intangibles no supone necesariamente más actividades innovadoras, entendemos que la correlación entre estos dos conceptos es evidente, por lo que podemos tomar la inversión en activos intangibles como una aproximación o sinónimo de emprendimiento e innovación. 
Por ello, en el estudio empírico que realizamos para las cooperativas hemos tratado de encontrar diferencias en rentabilidad, solvencia, endeudamiento, fiscalidad y empleabilidad entre las empresas cooperativas (no financieras) consideradas innovadoras y las clasificadas como no innovadoras, realizando un análisis estadístico de sus datos financieros para el período 2009-2011. Las cooperativas con inversión en intangibles superior a la media son consideradas como innovadoras, mientras que las que están por debajo de la media se las encuadra en el grupo de no innovadoras.

\section{4.- La contabilización del inmovilizado intangible en el PGC}

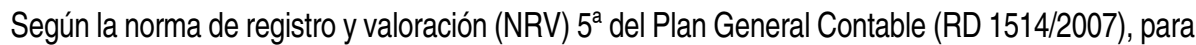
poder reconocer un inmovilizado de naturaleza intangible se debe cumplir con el "criterio de identificabilidad" que supone que pueda ser separado de la empresa (vendido, cedido, arrendado o intercambiado) o que surja de derechos legales o contractuales, es decir, que se haya pagado por su obtención. Así, no se reconocerán en ningún caso como inmovilizados intangibles los gastos ocasionados con motivo del establecimiento, las marcas, cabeceras de periódicos o revistas, los sellos 0 denominaciones editoriales, las listas de clientes u otras partidas similares que se hayan generado internamente.

Para su valoración posterior la empresa debe apreciar si la vida útil del inmovilizado intangible es definida 0 indefinida (sobre la base de todos los factores relevantes no hay un límite previsible del período a lo largo del cual se espera que el activo genere entradas de flujos netos de efectivo para la empresa) no amortizándose en este último caso aunque sí que deberá analizarse al menos anualmente su eventual deterioro.

Además, se establecen unas normas particulares sobre el inmovilizado intangible (NRV $6^{\mathrm{a}}$ ):

a) Investigación y desarrollo: serán gastos del ejercicio en que se realicen aunque podrán activarse como inmovilizado intangible cuando están específicamente individualizados por proyectos y su coste claramente establecido para que pueda ser distribuido en el tiempo además de tener motivos fundados de éxito técnico y de la rentabilidad económico-comercial del proyecto o proyectos de que se trate

Los gastos de investigación activados deberán amortizarse durante su vida útil dentro del plazo de cinco años y en el caso de que existan dudas razonables sobre el éxito técnico o la rentabilidad económico-comercial del proyecto deberán imputarse como gasto. Las condiciones para la activación de los gastos de desarrollo son similares a las establecidas para los gastos de establecimiento. 
b) Propiedad industrial: recogerá los gastos de desarrollo capitalizados cuando se obtenga la correspondiente patente 0 similar, incluyendo los costes de registro y formalización así como los importes pagados a terceros por la adquisición de los derechos correspondientes. Su amortización y deterioro debe contabilizarse según lo establecido con carácter general para los inmovilizados intangibles.

c) Fondo de comercio: sólo podrá figurar en el activo cuando su valor se ponga de manifiesto en virtud de una adquisición onerosa en el contexto de una combinación de negocios. No se amortizará, comprobándose cada año su posible deterioro de valor.

d) Derechos de traspaso: figurarán en el activo cuando se adquieran onerosamente, amortizándose y deteriorándose según el criterio general.

e) Programas informáticos: se incluyen tanto los adquiridos a terceros como los elaborados por la propia empresa para sí misma y los gastos de desarrollo de las páginas web. En ningún caso podrán figurar los gastos de mantenimiento de la aplicación informática. La amortización seguirá los criterios establecidos para los gastos de desarrollo y las correcciones valorativas por deterioro el criterio general.

f) Otros inmovilizados intangibles: también serán reconocidos como tales en el balance las concesiones administrativas, derechos comerciales, propiedad intelectual o licencias que se amortizarán y deteriorarán según el criterio general.

Como se aprecia en el detalle del inmovilizado intangible que se acaba de recoger, la mayoría de partidas incluidas en este epígrafe del balance y, sobre todo, las que más protagonismo pueden tener en las empresas cooperativas, guardan una relación evidente con la innovación. Por ello, un aumento de sus importes supone normalmente una apuesta por el emprendimiento y el desarrollo de las tecnologías de la información y comunicación. En este sentido, pues, podemos considerar el inmovilizado intangible como un proxy o indicador de la innovación.

\section{5.- Selección y fuentes de la muestra de empresas}

En el proceso de selección y obtención de las diferentes muestras de empresas se ha recurrido a la base de datos financieros $S A B I$ (Sistema de Análisis de Balances lbéricos), cuyo uso ha sido cedido a la Universitat de València por la empresa INFORMA S.A. 
Para seleccionar las empresas que formarían parte de la muestra de estimación el estudio se limitaría a aquellas cooperativas activas entre los años 2009 y 2011 de naturaleza no financiera, eliminando las de seguros y las crediticias. La selección efectuada, considerando esta condición, ofreció una cifra de 4.775 empresas cooperativas activas distribuidas en diferentes tamaños, sectores y zonas geográficas en el período considerado.

En estas empresas se realizó un filtrado para depurar aquellas empresas que presentaban datos incompletos en alguno de los ejercicios del periodo de estudio considerado. También se descartaron aquellas empresas de reciente creación (hasta tres años).

Tras estos procesos de selección y filtrado, el número de firmas se quedó en 1.991 empresas cooperativas que son las que definitivamente se integrarán en la muestra de estudio.

Para clasificar la muestra de trabajo atendiendo al tamaño de las empresas que la forman se ha tomado como criterio el número de trabajadores ${ }^{1}$ que conforman sus plantillas. Con la aplicación de este criterio la distribución por tamaños queda como se muestra en la Tabla 1:

\section{Tabla 1. Distribución por tamaños de las empresas cooperativas}

\begin{tabular}{|lc|}
\hline TAMAÑO & TOTALES \\
\hline GRANDES & 51 \\
MEDIANAS & 119 \\
PEQUEÑAS & 420 \\
MICROEMPRESAS & 1.401 \\
TOTAL & 1.991 \\
\hline
\end{tabular}

FUENTE: SABI.

En la Tabla 2 se presenta el resumen de la distribución de la muestra por sectores de actividad económica mostrando el peso específico de cada grupo sectorial en el total de la muestra. La agrupación por sectores se ha realizado atendiendo al C.N.A.E. de 2009 considerando los dos primeros dígitos de control, lo que ha permitido distinguir 14 actividades básicas.

1.- Criterios cuantitativos para la clasificación de empresas

\begin{tabular}{|l|cccc|}
\hline CRITERIOS & $\begin{array}{c}\text { EMPRESA } \\
\text { GRANDE }\end{array}$ & $\begin{array}{c}\text { EMPRESA } \\
\text { MEDIANA }\end{array}$ & $\begin{array}{c}\text { EMPRESA } \\
\text { PEQUEÑA }\end{array}$ & $\begin{array}{c}\text { MICRO } \\
\text { EMPRESA }\end{array}$ \\
\hline PLANTILLA DE LA EMPRESA & $>250$ & $<250$ & $<50$ & $<10$ \\
\hline
\end{tabular}

FUENTE: Comisión de las Comunidades Europeas. 


\section{Tabla 2. Distribución de la muestra de empresas por} sectores de actividad económica

\begin{tabular}{|llc|}
\hline CNAE-2009 & ACTIVIDAD & No EMPRESAS \\
\hline $01-03$ & Agricultura, ganadería, selvicultura y pesca & 516 \\
$05-09$ & Industrias extractivas & 3 \\
$10-33$ & Industria manufacturera & 357 \\
35 & Suministro de energía & 6 \\
$36-39$ & Suministro de agua & 20 \\
$41-43$ & Construcción & 167 \\
$45-47$ & Comercio al por mayor y al por menor & 572 \\
$49-53$ & Transportes & 89 \\
$55-56$ & Hosteleria & 20 \\
$58-63$ & Información y comunicaciones & 17 \\
$64-66$ & Actividades financieras y seguros & 10 \\
$68-75$ & Actividades inmobiliarias & 75 \\
$77-82$ & Actividades administrativas y servicios aux. & 45 \\
& Resto & 94 \\
\hline TOTAL & & 1.991 \\
\hline
\end{tabular}

FUENTE: Elaboración propia.

Por último, en la Tabla 3 se presenta el resumen de la distribución de la muestra de empresas por comunidades autónomas.

\section{Tabla 3. Distribución de muestra de empresas por CC.AA.}

\begin{tabular}{|lc|}
\hline COMUNIDAD & No EMPRESAS $^{\circ}$ \\
\hline Andalucía & 181 \\
Aragón & 238 \\
Asturias & 70 \\
Baleares & 4 \\
Canarias & 38 \\
Castilla y León & 121 \\
Castilla-La Mancha & 203 \\
Cataluña & 61 \\
Comunidad Valenciana & 441 \\
Extremadura & 310 \\
Galicia & 48 \\
La Rioja & 16 \\
Madrid & 32 \\
Melilla & 12 \\
Murcia & 50 \\
Navarra & 20 \\
País Vasco & 146 \\
\hline & 1.991 \\
\hline
\end{tabular}

FUENTE: Elaboración propia. 
De la interpretación de los datos que se muestran en las anteriores tablas deducimos la correcta representatividad de la muestra confeccionada para llevar a cabo nuestro estudio.

\subsection{Selección y definición de las variables explicativas}

Para seleccionar los ratios con los que comenzaremos a trabajar nos basaremos en la facilidad para ser calculados y definidos en función de la información contable disponible.

En principio consideramos una amplia lista de ratios contenidos en diferentes categorías. Los solapamientos de información y problemas estadísticos, que necesariamente han de producirse, serán depurados con las técnicas estadísticas apropiadas eliminando aquellos que apenas aporten información adicional. Incidiremos en aquellas variables que, en principio, informen sobre los aspectos de la solvencia y rentabilidad de la firma, sin olvidar la influencia del endeudamiento. A estas categorías añadiremos las de rotación, actividad y estructura de activo. Describimos a continuación las categorías en las que incluiremos los diferentes ratios:

\section{Rentabilidad (REN)}

Genéricamente la rentabilidad se identifica con el cociente entre beneficio e inversión y es la tasa con que la empresa remunera al capital empleado. La rentabilidad es una medida del rendimiento empresarial. Dependiendo del tipo de beneficio específico considerado, que figurará en el numerador de los ratios propuestos y de la magnitud que lo ha generado, representada en el denominador, tendremos diversos tipos de rentabilidad, económica y financiera. Existe prácticamente unanimidad en la doctrina de análisis contable en considerar la rentabilidad económica como indicador de la eficacia de la empresa en la utilización de sus inversiones y la rentabilidad financiera como indicador de la capacidad de la empresa para crear riqueza a favor de sus propietarios. Sin embargo, no debemos olvidar que en las cooperativas la mejor rentabilidad de sus socios vendrá probablemente por la obtención de unos buenos precios de los productos y servicios cooperativizados.

\section{Estructura financiera o Endeudamiento (EF)}

La estructura financiera informa sobre el origen y composición de los recursos financieros que han servido para llevar a cabo la inversión que se ha materializado en el conjunto de factores de producción a disposición de la empresa o estructura económica. Pero tal y como hemos indicado en las especificidades de las cooperativas, nos encontramos con una magnitud, el capital, que puede haberse considerado patrimonio neto cuando en el fondo puede ser un pasivo, al menos en parte.

Con estos indicadores se podrá establecer el grado de dependencia de la empresa en función de la fuente de recursos financieros que utiliza. 


\section{ANÁLISIS DE LA INNOVACIÓN EN LAS COOPERATIVAS MEDIANTE LA CAPTACIÓN DEL VALOR INTANGIBLE: UN ESTUDIO EMPÍRICO}

\section{Solvencia a corto plazo (SOLV)}

Los ratios de solvencia a corto plazo o liquidez, ligados estrechamente a la situación de equilibrio financiero, indican la capacidad que la empresa tiene para atender sus deudas a corto plazo (por corto plazo entenderemos el ejercicio económico o el ciclo de explotación si este es superior a un año). Estos ratios relacionan partidas o masas patrimoniales de la estructura circulante de la empresa, tanto del activo como del pasivo. Nos servirán para determinar la liquidez del activo.

\section{Solvencia a largo plazo (SOLV LP)}

Con estos ratios se pretende determinar la capacidad de la empresa para afrontar sus deudas a largo plazo. Estas medidas centran su atención en la estructura financiera y operativa de la empresa contemplada desde el largo plazo.

\section{Variación (VAR)}

Con ellos se pretende analizar la variación y evolución de ciertas magnitudes en el periodo de estudio considerado.

\section{Ratio de fiscalidad (FIS)}

Mediremos la proporción que supone el gasto contable por impuesto sobre sociedades sobre el resultado contable antes de impuestos.

En nuestro estudio partiremos de la información contenida en los estados financieros de las sociedades cooperativas transformándola en ratios, lo que nos permitirá establecer con rigor su situación económico-financiera y realizar comparaciones entre las distintas empresas y diferentes periodos económicos. Por razones de tipo operativo no hemos considerado información de distinto origen, como la de naturaleza cualitativa.

La lista de los ratios considerados inicialmente y separados por categorías la mostramos en la Tabla 4: 
Tabla 4. Ratios utilizados en el estudio empírico

\begin{tabular}{|c|c|c|}
\hline \multicolumn{3}{|c|}{ RENTABILIDAD } \\
\hline CLAVE & RATIO & \\
\hline REN 1 & RESULTADO DE EXPLOTACION/ACTIVO TOTAL & RE/AT \\
\hline REN 2 & RESULTADO ANTES DE IMPUESTOS / FONDOS PROPIOS & $\mathrm{RAl} / \mathrm{FP}$ \\
\hline REN 3 & RESULTADO DEL EJERCICIO/FONDOS PROPIOS & R/FP \\
\hline \multicolumn{3}{|c|}{ ENDEUDAMIENTO } \\
\hline CLAVE & RATIO & \\
\hline END 1 & PASIVO TOTAL / FONDOS PROPIOS & PT/FP \\
\hline END 2 & GASTOS FINANCIEROS / PASIVO TOTAL & GF/PT \\
\hline END 3 & PASIVO FIJO/FONDOS PROPIOS & PF/FP \\
\hline END 4 & GASTOS FINANCIEROS / VENTAS & GF/V \\
\hline END 5 & PASIVO CORRIENTE / PASIVO NO CORRIENTE & PC/PNC \\
\hline
\end{tabular}

\begin{tabular}{|clc|}
\hline \multicolumn{2}{c|}{ SOLVENCIA (Liquidez) } \\
\hline CLAVE & \multicolumn{1}{c|}{ RATIO } \\
\hline SOLV 1 & ACTIVO CORRIENTE / PASIVO CORRIENTE & AC/PC \\
SOLV 2 & ACTIVO CIRCULANTE - EXISTENCIAS / PASIVO CORRIENTE & AC-EX/PC \\
SOLV 3 & DISPONIBLE/PASIVO CORRIENTE & D/PC \\
SOLV 4 & CAPITAL CORRIENTE / PASIVO CORRIENTE & CC/PC \\
SOLV 5 & ACTIVO CIRCULANTE - EXISTENCIAS/VENTAS & AC-EX/V \\
\hline
\end{tabular}

\begin{tabular}{|llc|}
\hline \multicolumn{3}{|c|}{ SOLVENCIA A LARGO PLAZO } \\
\hline CLAVE & \multicolumn{1}{c|}{ RATIO } \\
\hline SOLV LP 1 & RECURSOS GENERADOS ANTES DE IMPUESTOS/PASIVO TOTAL & RGAI/PT \\
SOLV LP 2 & ACTIVO TOTAL/PASIVO TOTAL & AT/PT \\
\hline
\end{tabular}

\begin{tabular}{|ll|}
\hline \multicolumn{1}{|c|}{ RATIOS DE CRECIMIENTO } \\
\hline VAR. VENTAS & \multicolumn{1}{c|}{ VENTAS (N) - VENTAS (N-1) / VENTAS (N-1) } \\
VAR. TRABAJ. & $\mathrm{N}^{\circ}$ TRABAJADORES (N) - No DE TRABAJADORES (N-1) / N ${ }^{\circ}$ DE TRABAJADORES (N-1) \\
VAR. ACTIVO & ACTIVO (N) - ACTIVO (N-1) / ACTIVO (N-1) \\
\hline
\end{tabular}

\begin{tabular}{|clc|}
\hline \multicolumn{3}{|c|}{ FISCALIDAD } \\
\hline FIS 1 & IMPUESTO DE SOCIEDADES / RDO. ANTES DE IMPUESTOS & IS/RAI \\
FIS 2 & IMPUESTO DE SOCIEDADES / RESULTADO DEL EJERCICIO & IS/RDI \\
\hline
\end{tabular}

FUENTE: Elaboración propia.

Todas las partidas integrantes de los ratios han sido derivadas del Balance de Situación y cuenta de Pérdidas y Ganancias de las empresas que componen la muestra. 
El número de observaciones que aparecen en las tablas del epígrafe siguiente de resultados no son coincidentes debido a la presencia de diferentes valores extremos y valores perdidos para cada tipo de análisis efectuado, que han sido eliminados tanto para el estudio descriptivo como estadístico.

\section{6.- Resultados}

\subsection{Análisis de rentabilidad}

Antes de proceder con el análisis de rentabilidad de las empresas cooperativas atendiendo al factor innovación, se ha comprobado que no hubiese problemas de multicolinealidad y correlación ${ }^{2}$ entre las variables mediante la matriz de los coeficientes de correlación de Pearson. Dado que no hemos observado correlaciones altas entre estos coeficientes, en el análisis que realizaremos consideraremos todos los ratios propuestos.

La Tabla 5 resume los principales estadísticos descriptivos de las variables relacionadas con la rentabilidad. Se observa que dependiendo del ratio la media es mayor en una de las dos categorías de cooperativas clasificadas según el criterio de innovación. La Figura 3 recoge los resultados del estudio de los perfiles que toman los ratios de rentabilidad de acuerdo con la variable de agrupación, esto es, de los dos grupos estudiados. Ninguna de las dos líneas predomina sobre la otra y de hecho como se aprecia se entrecruzan, por lo que el análisis exploratorio no nos muestra claramente la existencia de diferencias entre estos dos grupos de sociedades cooperativas en lo que a rentabilidad se refiere.

\section{Tabla 5. Resumen de los estadísticos descriptivos. Rentabilidad}

\begin{tabular}{|lrrrrr|}
\hline No innovadoras & \multicolumn{5}{c|}{} \\
\hline Variable & Obs. & Media & D. Estándar & Mín. & Máx. \\
\hline ren1 & 1.705 & $-0,013$ & 0,139 & $-2,524$ & 0,974 \\
ren2 & 1.705 & 0,037 & 0,769 & $-5,911$ & 9,069 \\
ren3 & 1.705 & 0,026 & 0,68 & $-4,839$ & 9,069 \\
\hline Innovadoras & & & & & \\
\hline Variable & Obs. & Media & D. Estándar & Mín. & Máx. \\
\hline ren1 & 251 & 0.004 & 0.126 & -1.476 & 0.544 \\
ren2 & 251 & 0.003 & 0.593 & -3.386 & 3.249 \\
ren3 & 251 & -0.001 & 0.537 & -3.386 & 3.174 \\
\hline
\end{tabular}

2.- Por motivos de espacio no hemos incluido en el trabajo ninguna matriz de correlaciones. 


\section{Figura 3. Gráfico de perfil de las medias. Rentabilidad}

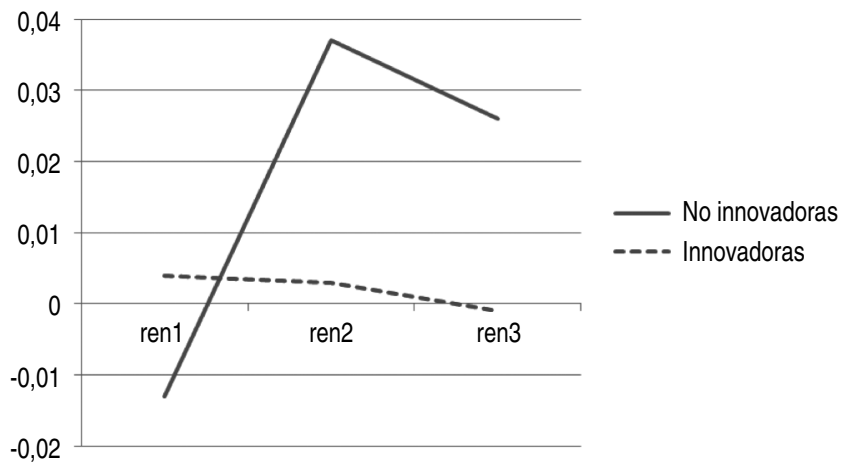

Con el fin de identificar la presencia de diferencias que no han podido ser puestas de manifiesto a partir de las herramientas descriptivas comentadas, nos hemos apoyado en distintas pruebas estadísticas multivariantes y univariantes. Ninguno de los contrastes multivariantes realizados (Tabla 6), basados en el modelo lineal general MANOVA, resulta estadísticamente significativo. Por consiguiente, cuando se mide la rentabilidad con los ratios señalados de forma agregada ésta no difiere entre las sociedades cooperativas innovadoras y no innovadoras.

Mediante el análisis de varianza univariante (ANOVA) se ha comprobado si alguna variable en particular, tomada ahora por separado, muestra alguna diferencia relevante entre las dos categorías analizadas. En la Tabla 7 se aprecia que la variable ren1 es significativa a un nivel del 10,00\%. La relación que contiene este ratio, resultado de explotación y activo total, es mayor para el grupo de las sociedades cooperativas innovadoras tal y como se aprecia en el análisis exploratorio comentado.

\section{Tabla 6. Pruebas multivariantes. Ratios de rentabilidad}

\begin{tabular}{|llccccc|}
\hline Efecto & & Valor & Grados hipótesis & Grados error & $\boldsymbol{F}$ & Sig. \\
\hline Innovación & Lambda de Wilks & 0,9981 & 3 & 1952 & 1,26 & 0,2861 \\
& Traza de Pillai & 0,0019 & 3 & 1952 & 1,26 & 0,2861 \\
& Traza de Hotelling & 0,0019 & 3 & 1952 & 1,26 & 0,2861 \\
& Raiz mayor de Roy & 0,0019 & 3 & 1952 & 1,26 & 0,2861 \\
\hline
\end{tabular}




\section{Tabla 7. Pruebas univariantes. Ratios de rentabilidad}

\begin{tabular}{|cccc|}
\hline Ratio & Medias cuadradas & $\boldsymbol{F}$ & Significat. \\
\hline ren1 & 0,01887 & 3,21 & 0,0733 \\
ren2 & 0,56088 & 0,45 & 0,5037 \\
ren3 & 0,43956 & 0,37 & 0,5458 \\
\hline
\end{tabular}

\subsection{Análisis del endeudamiento}

La Tabla 8 muestra un resumen de los estadísticos descriptivos de las variables empleadas para comparar el efecto de la innovación sobre el endeudamiento. En principio, se observa que los valores de endeudamiento para los distintos ratios son generalmente mayores en las cooperativas consideradas como innovadoras. La Figura 4 que recoge el perfil de la línea de los valores de ambos grupos también está en consonancia con estos resultados, aunque no está del todo claro ya que el trazado o perfil de la línea de las innovadoras no discurre siempre por encima del de las no innovadoras. Ante esta falta de claridad en los resultados del análisis descriptivo resulta imprescindible realizar el análisis estadístico. La Tabla 9 muestra que ninguno de los contrastes multivariados (MANOVA) de los ratios de endeudamiento es significativo incluso a un nivel del 10,00\%. Por consiguiente, de acuerdo con el efecto conjunto de todos estos indicadores, el nivel de endeudamiento no difiere significativamente entre las cooperativas innovadoras y las no innovadoras.

Por otro lado, mediante tests ANOVA univariantes, se ha contrastado si existen diferencias entre estos ratios cuando se analizan por separado. Ninguno de los ratios considerados resulta estadísticamente relevante (Tabla 10), concluyendo que el factor innovación no ejerce un efecto diferenciador en el endeudamiento de las sociedades cooperativas. Sin embargo, los resultados del estudio descriptivo, como se ha indicado, reflejan que el endeudamiento en general es ligeramente mayor en las sociedades cooperativas innovadoras. Hay que tener en cuenta que las empresas más innovadoras pueden tener intangibles que no están reconocidos contablemente como sería el caso de un fondo de comercio no adquirido, lo que repercutirá negativamente en sus indicadores de endeudamiento. 


\section{Tabla 8. Resumen de los estadísticos descriptivos.} Endeudamiento

\begin{tabular}{|lrrrrr|}
\hline No innovadoras & \multicolumn{5}{c|}{} \\
\hline Variable & Obs. & Media & D. Estándar & Mín. & Máx. \\
\hline end1 & 1.497 & 4,591 & 28,725 & $-261,432$ & 617,491 \\
end2 & 1.497 & 0,024 & 0,029 & 0,001 & 0,347 \\
end3 & 1.497 & 0,839 & 3,474 & $-17,892$ & 49,172 \\
end4 & 1.497 & 0,038 & 0,313 & 0,001 & 11,439 \\
end5 & 1.012 & 71,646 & $1.013,099$ & 0,008 & $29.070,01$ \\
\hline Innovadoras & & & & Mín. \\
\hline Variable & Obs. & Media & D. Estándar & Máx. \\
\hline end1 & 230 & 4,326 & 10,907 & $-21,601$ & 114,653 \\
end2 & 230 & 0,026 & 0,021 & 0,001 & 0,169 \\
end3 & 230 & 1,178 & 3,735 & $-13,17$ & 25,662 \\
end4 & 230 & 0,023 & 0,04 & 0,001 & 0,366 \\
end5 & 186 & 197,797 & $1.715,817$ & 0,032 & $16.969,48$ \\
\hline
\end{tabular}

Figura 4. Gráfico de perfil de las medias. Endeudamiento

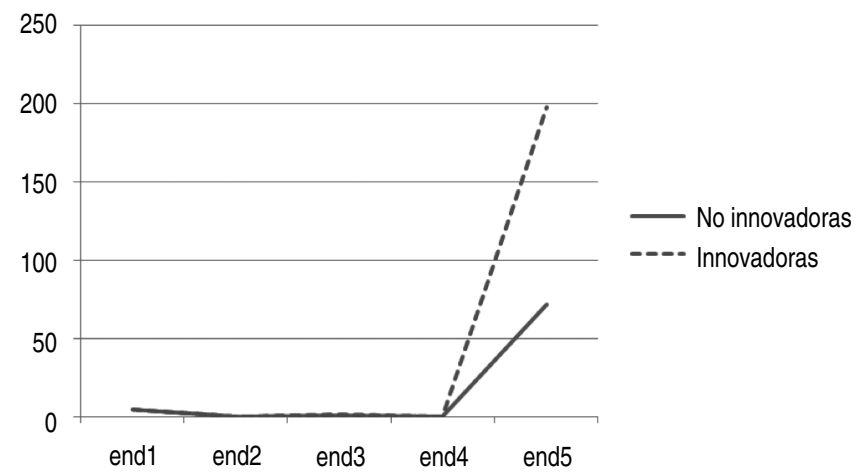




\section{Tabla 9. Pruebas multivariantes. Ratios de endeudamiento}

\begin{tabular}{|llccccc|}
\hline Efecto & & Valor & Grados hipótesis & Grados error & $\boldsymbol{F}$ & Sig. \\
\hline Innovación & Lambda de Wilks & 0,9958 & 5 & 1192 & 1,01 & 0,4078 \\
& Traza de Pillai & 0,0042 & 5 & 1192 & 1,01 & 0,4078 \\
& Traza de Hotelling & 0,0043 & 5 & 1192 & 1,01 & 0,4078 \\
& Raiz mayor de Roy & 0,0043 & 5 & 1192 & 1,01 & 0,4078 \\
\hline
\end{tabular}

\section{Tabla 10. Pruebas univariantes. Ratios de endeudamiento}

\begin{tabular}{|lrcc|}
\hline Ratio & Medias cuadradas & $\boldsymbol{F}$ & Significat. \\
\hline end1 & 730,9422 & 0,02 & 0,8901 \\
end2 & 0,0007 & 0,98 & 0,3212 \\
end3 & 12,3249 & 1,87 & 0,1716 \\
end4 & 0,0851 & 0,48 & 0,4868 \\
end5 & $1.323 .980,57$ & 1,89 & 0,1695 \\
\hline
\end{tabular}

\subsection{Análisis de la solvencia}

La matriz de correlaciones ha desvelado la presencia de problemas de multicolinealidad en los ratios de esta categoría propuestos inicialmente por lo que nos hemos visto obligados a eliminar la variable solv4. Los principales estadísticos descriptivos del resto de variables empleadas para comparar la solvencia se muestran en la Tabla 11. Se aprecia que los valores más altos de la media de estos ratios predominan en el segundo grupo, como se deriva de su representación gráfica (Figura 5). Por tanto, en principio habría discrepancias en la valoración de la solvencia entre estos dos grupos de cooperativas.

Para confirmar la existencia de tales diferencias nos hemos valido de nuevo del análisis estadístico. Ninguno de los contrastes multivariados de la Tabla 12 de los ratios que definen la solvencia es significativo a niveles del $10,00 \%$. Por consiguiente, la solvencia cuando se mide tomando el efecto conjunto de todos estos indicadores, esto es, de acuerdo con las pruebas MANOVA, es similar entre las cooperativas innovadoras y las no innovadoras.

Para contrastar si existen diferencias entre estos ratios cuando se analizan por separado hemos empleado pruebas ANOVA univariantes. Los resultados muestran que a un nivel del $5,00 \%$ el ratio de solvencia solv5 es estadísticamente significativo (Tabla 13). Dada su formulación, el menor valor para las cooperativas innovadoras podría responder a que éstas tienen un mayor volumen de ventas en 
comparación con las cooperativas no innovadoras. Estas conclusiones se verían respaldadas por los resultados obtenidos más adelante en el análisis del crecimiento, donde se observa que la cifra de negocios en las cooperativas innovadoras es mayor que en las no innovadoras.

\section{Tabla 11. Resumen de los estadísticos descriptivos. Solvencia}

\begin{tabular}{|lrrrrr|}
\hline No innovadoras & \multicolumn{5}{c|}{} \\
\hline Variable & Obs. & Media & D. Estándar & Mín. & Máx. \\
\hline solv1 & 1677 & 3,663 & 9,031 & 0,010 & 95,392 \\
solv2 & 1677 & 2,88 & 7,727 & 0,006 & 95,392 \\
solv3 & 1650 & 1,173 & 4,005 & 0,001 & 72,22 \\
solv5 & 1630 & 0,836 & 2,473 & 0,007 & 51,153 \\
\hline Innovadoras & & & & Mín. & Máx. \\
\hline Variable & Obs. & Media & D. Estándar & 0,051 & 59,841 \\
\hline solv1 & 252 & 3,134 & 7,284 & 0,051 & 58,963 \\
solv2 & 2,653 & 7,024 & 0,001 & 42,436 \\
solv3 & 252 & 1,148 & 4,174 & 0,030 & 6,644 \\
solv5 & 246 & 0,525 & 0,759 & \\
\hline
\end{tabular}

Figura 5. Gráfico de perfil de las medias. Solvencia

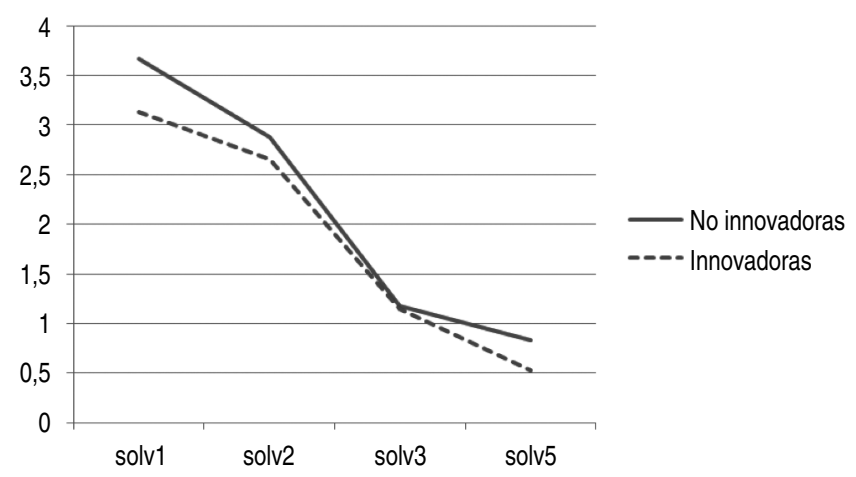




\section{Tabla 12. Pruebas multivariantes. Ratios de solvencia}

\begin{tabular}{|llccccc|}
\hline Efecto & & Valor & Grados hipótesis & Grados error & $\boldsymbol{F}$ & Sig. \\
\hline Innovación & Lambda de Wilks & 0,9971 & 4 & 1815 & 1,33 & 0,2552 \\
& Traza de Pillai & 0,0029 & 4 & 1815 & 1,33 & 0,2552 \\
& Traza de Hotelling & 0,0029 & 4 & 1815 & 1,33 & 0,2552 \\
& Raiz mayor de Roy & 0,0029 & 4 & 1815 & 1,33 & 0,2552 \\
\hline
\end{tabular}

\section{Tabla 13. Pruebas univariantes. Ratios de solvencia}

\begin{tabular}{|cccc|}
\hline Ratio & Medias cuadradas & $\boldsymbol{F}$ & Significat. \\
\hline solv1 & 77,8400 & 0,79 & 0,3753 \\
solv2 & 58,3280 & 0,19 & 0,6606 \\
solv3 & 16,2106 & 0,01 & 0,9284 \\
solv5 & 5,4114 & 3,78 & 0,0520 \\
\hline
\end{tabular}

\subsection{Análisis de la solvencia a largo plazo}

En este apartado analizamos la solvencia a largo plazo, cuyos estadísticos descriptivos de las variables podemos ver en la Tabla 14. Observamos que, dependiendo del ratio, el valor de la media es mayor en un tipo $u$ otro de sociedades cooperativas. Además, en la Figura 6 podemos ver que las líneas que representan los valores de ambas categorías de cooperativas se entrecruzan, aunque se aprecia que el ratio solvip2 en las cooperativas no innovadoras está por encima de las innovadoras.

El estudio estadístico de los ratios de solvencia a largo plazo nos ayudará a determinar si efectivamente hay diferencias en las cooperativas innovadoras y no innovadoras. De acuerdo con el análisis multivariante (MANOVA), el efecto global de los dos ratios de la solvencia a largo plazo examinados no difiere significativamente entre estos dos grupos de sociedades (Tabla 15).

Con el análisis univariante (ANOVA) identificamos si algún ratio en particular resulta estadísticamente significativo. En la Tabla 16 se aprecia que, a un nivel del 5,00\%, la solvencia a largo plazo medida con el ratio solvip2 es significativamente mayor para las cooperativas no innovadoras. Considerando las magnitudes que lo forman (cociente entre los activos totales y el pasivo total) deduciríamos que las empresas innovadoras podrían tener unos mayores pasivos consecuencia de un mayor endeudamiento para hacer frente a las actividades relacionadas con la innovación. En el análisis del endeudamiento se ha observado en este sentido un mayor endeudamiento precisamente en las cooperativas clasificadas como innovadoras, aunque no resultase estadísticamente significativo. 
Tabla 14. Resumen de los estadísticos descriptivos. Solvencia a largo plazo

\begin{tabular}{|lccrrr|}
\hline No innovadoras & \multicolumn{5}{c|}{} \\
\hline Variable & Obs. & Media & D. Estándar & Mín. & Máx. \\
\hline solv1p1 & 1696 & $-0,023$ & 0,894 & $-23,534$ & 6,714 \\
solvlp2 & 1696 & 4,441 & 12,855 & 0,020 & 183,818 \\
\hline Innovadoras & & & & & \\
\hline Variable & Obs. & Media & D. Estándar & Mín. & Máx. \\
\hline solvlp1 & 253 & 0,033 & 0,45 & $-3,601$ & 4,022 \\
solvlp2 & 253 & 2,828 & 5,865 & 0,320 & 78,198 \\
\hline
\end{tabular}

Figura 6. Gráfico de perfil de las medias. Solvencia a largo plazo

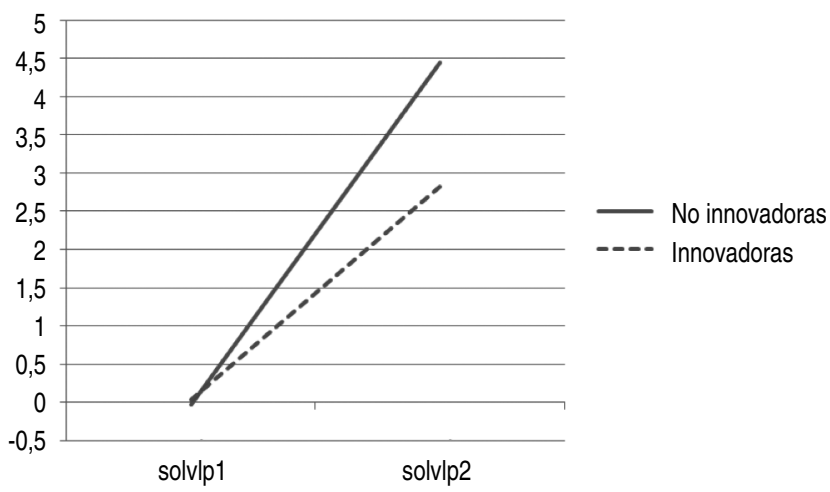

\section{Tabla 15. Pruebas multivariantes. Ratios de solvencia a largo plazo}

\begin{tabular}{|llccccc|}
\hline Efecto & & Valor & Grados hipótesis & Grados error & $\boldsymbol{F}$ & Sig. \\
\hline Innovación & Lambda de Wilks & 0,9979 & 2 & 1946 & 2,05 & 0,1295 \\
& Traza de Pillai & 0,0021 & 2 & 1946 & 2,05 & 0,1295 \\
& Traza de Hotelling & 0,0021 & 2 & 1946 & 2,05 & 0,1295 \\
& Raiz mayor de Roy & 0,0021 & 2 & 1946 & 2,05 & 0,1295 \\
\hline
\end{tabular}




\section{Tabla 16. Pruebas univariantes. Ratios de solvencia a largo plazo}

\begin{tabular}{|lccc|}
\hline Ratio & Medias cuadradas & $\boldsymbol{F}$ & Significat. \\
\hline solvlp1 & 0,7225 & 0,97 & 0,3254 \\
solvlp2 & 148,5306 & 3,86 & 0,0496 \\
\hline
\end{tabular}

\subsection{Análisis de la fiscalidad}

Un resumen de los estadísticos descriptivos de las variables empleadas para comparar la fiscalidad entre los dos grupos de empresas considerados se muestra en la Tabla 17. En principio se observa que los valores de la carga fiscal en los dos ratios analizados son menores en las cooperativas innovadoras. La Figura 7, que recoge el perfil de la línea que dibuja los valores de ambos grupos, también está en consonancia con estos resultados, y el trazado de la línea de las innovadoras discurre por debajo de las no innovadoras.

\section{Tabla 17. Resumen de estadísticos descriptivos. Fiscalidad}

\begin{tabular}{|lcrrrr|}
\hline No innovadoras & \multicolumn{5}{c|}{} \\
\hline Variable & Obs. & Media & D. Estándar & Mín. & Máx. \\
\hline fisc1 & 1698 & 0,136 & 0,389 & $-13,389$ & 3,382 \\
fisc2 & 1697 & 0,254 & 1,179 & $-8,172$ & 31,262 \\
\hline Innovadoras & & & & \\
\hline Variable & Obs. & Media & D. Estándar & Mín. & Máx. \\
\hline fisc1 & 251 & 0,110 & 0,483 & $-6,025$ & 3,951 \\
fisc2 & 251 & 0,204 & 0,404 & $-0,523$ & 4,183 \\
\hline
\end{tabular}

\section{Figura 7. Gráfico de perfil de las medias. Fiscalidad}

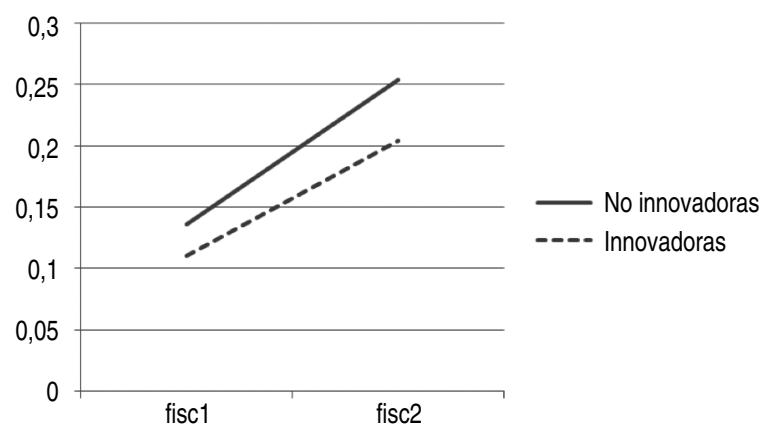


El análisis estadístico del comportamiento multivariante (MANOVA) de las variables empleadas para medir la presión fiscal muestra que ninguno de los contrastes resulta estadísticamente significativo (Tabla 18). Por consiguiente, de acuerdo con el efecto conjunto de todos estos indicadores, la carga fiscal no diferiría entre las cooperativas innovadoras y las no innovadoras.

Por otro lado, mediante test ANOVA univariantes se ha contrastado si existen desigualdades entre estos ratios cuando se analizan por separado. Se observa que las diferencias apreciadas resultan estadísticamente significativa (Tabla 19).

En resumen, los resultados de las pruebas univariantes y multivariantes indican que la fiscalidad no difiere entre los dos grupos de cooperativas analizadas. Sin embargo, el estudio descriptivo revela que la media de la carga fiscal es menor para las cooperativas innovadoras, entre 2 y 5 puntos enteros dependiendo del ratio.

\section{Tabla 18. Pruebas multivariantes. Ratios de fiscalidad}

\begin{tabular}{|llccccc|}
\hline Efecto & & Valor & Grados hipótesis & Grados error & $\boldsymbol{F}$ & Sig. \\
\hline Innovación & Lambda de Wilks & 0,9994 & 2 & 1945 & 0,61 & 0,5418 \\
& Traza de Pillai & 0,0006 & 2 & 1945 & 0,61 & 0,5418 \\
& Traza de Hotelling & 0,0006 & 2 & 1945 & 0,61 & 0,5418 \\
& Raiz mayor de Roy & 0,0006 & 2 & 1945 & 0,61 & 0,5418 \\
\hline
\end{tabular}

\section{Tabla 19. Pruebas univariantes. Ratios de fiscalidad}

\begin{tabular}{|lccc|}
\hline Ratio & Medias cuadradas & $\boldsymbol{F}$ & Significat. \\
\hline fisc1 & 0,1617 & 0,93 & 0,3339 \\
fisc2 & 1,2319 & 0,45 & 0,5030 \\
\hline
\end{tabular}

\subsection{Análisis del crecimiento}

La Tabla 20 recoge el resumen de los principales estadísticos descriptivos de las variables relacionadas con el crecimiento tenidas en cuenta en este estudio. Se observa que la media es mayor en la categoría de las cooperativas clasificadas como innovadoras. La Figura 8 muestra los resultados del estudio de los perfiles que toman los ratios de crecimiento de acuerdo con la variable de agrupación innovación. Se aprecia claramente que una de las dos líneas predomina sobre la otra, por lo que el análisis exploratorio realizado, en principio, estaría evidenciando la existencia de diferencias entre los dos grupos de sociedades cooperativas en lo que a crecimiento se refiere, que sería superior para las que realizan actividades de innovación. 


\section{Tabla 20. Resumen de los estadísticos descriptivos. Crecimiento}

\begin{tabular}{|lccrrr|}
\hline No innovadoras & \multicolumn{5}{c|}{} \\
\hline Variable & Obs. & Media & D. Estándar & Mín. & Máx. \\
\hline crecimvtas & 1526 & 0,163 & 2,466 & $-0,867$ & 92,805 \\
crecimact & 1597 & 0,120 & 0,491 & $-0,633$ & 7,821 \\
\hline Innovadoras & & & & Mín. & Máx. \\
\hline Variable & Obs. & Media & D. Estándar & $-0,675$ & 222,192 \\
\hline crecimvtas & 226 & 1,040 & 14,779 & $-0,423$ & 148,947 \\
crecimact & 235 & 0,743 & 9,716 & &
\end{tabular}

\section{Figura 8. Gráfico de perfil de las medias. Crecimiento}

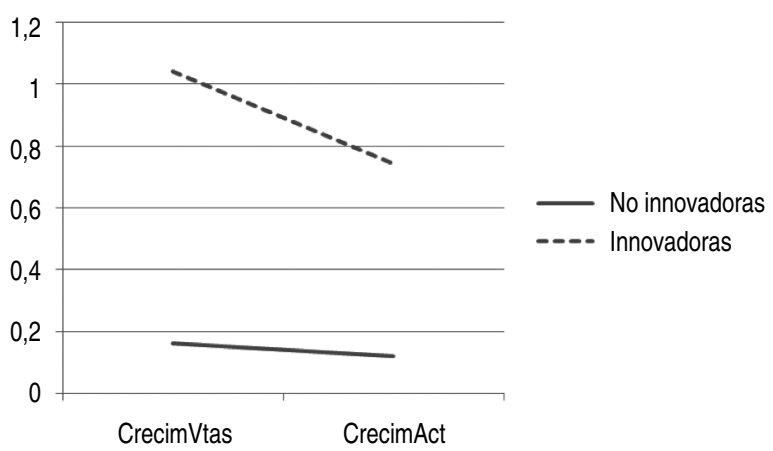

A continuación nos hemos apoyado en distintas pruebas estadísticas tanto multivariantes como univariantes para asegurarnos de la presencia de las diferencias puestas de manifiesto a partir de las herramientas descriptivas comentadas. Todos los contrastes multivariantes realizados (Tabla 21), basados en el modelo lineal general MANOVA, resultan estadísticamente significativos al 5,00\%. Por consiguiente, cuando se mide el crecimiento con los ratios señalados de forma agregada, las cooperativas clasificadas como innovadoras tienen mayores índices de crecimiento.

Mediante el análisis de varianza univariante (ANOVA) se ha comprobado cuál de las variables anteriores, tomada ahora por separado, muestra una diferencia relevante entre estas dos categorías de sociedades cooperativas. En la Tabla 22 se aprecia que las variables crecimvtas y crecimact son estadísticamente significativas a un nivel del $5,00 \%$ y $1,00 \%$ respectivamente, y mayores para las cooperativas innovadoras. 
Tabla 21. Pruebas multivariantes. Ratios de crecimiento

\begin{tabular}{|llccccc|}
\hline Efecto & & Valor & Grados hipótesis & Grados error & $\boldsymbol{F}$ & Sig. \\
\hline Innovación & Lambda de Wilks & 0,9959 & 2 & 1749 & 3,58 & 0,028 \\
& Traza de Pillai & 0,0041 & 2 & 1749 & 3,58 & 0,028 \\
& Traza de Hotelling & 0,0041 & 2 & 1749 & 3,58 & 0,028 \\
& Raiz mayor de Roy & 0,0041 & 2 & 1749 & 3,58 & 0,028 \\
\hline
\end{tabular}

\section{Tabla 22. Pruebas univariantes. Ratios de crecimiento}

\begin{tabular}{|lccc|}
\hline Ratio & Medias cuadradas & $\boldsymbol{F}$ & Significat. \\
\hline crecimvtas & 33,4504 & 4,54 & 0,0333 \\
crecimact & 12,3177 & 6,46 & 0,0111 \\
\hline
\end{tabular}

6.7. Análisis de la variación de la plantilla

Por último, hemos comprobado la existencia de diferencias entre las sociedades cooperativas innovadoras y las no innovadoras en cuanto a la variación media de la plantilla. Claramente se deduce de la Tabla 23 y de la Figura 9 que mientras en las sociedades no innovadoras la variación de la plantilla es prácticamente nula $(0,80 \%)$, en las innovadoras la media se ha incrementado en un $7,00 \%$.

\section{Tabla 23. Resumen de los estadísticos descriptivos. Plantilla}

\begin{tabular}{|lccrrr|}
\hline No innovadoras & \multicolumn{7}{c|}{} & \\
\hline Variable & Obs. & Media & D. Estándar & Mín. & Máx. \\
\hline VarTrab & 1111 & 0,008 & 0,193 & $-0,763$ & 1,035 \\
\hline Innovadoras & & & & \\
\hline Variable & Obs. & Media & D. Estándar & Mín, & Máx, \\
\hline VarTrab & 153 & 0,070 & 0,681 & $-0,367$ & 8 \\
\hline
\end{tabular}

Los resultados del análisis univariante confirman las conclusiones iniciales del estudio exploratorio. La variable considerada para medir la variación media de la plantilla es estadísticamente significativa a un nivel del $5,00 \%$ y muy superior, como se acaba de indicar, para las sociedades cooperativas clasificadas como innovadoras (ver Tabla 24). 


\section{Figura 9. Variación media de la plantilla}

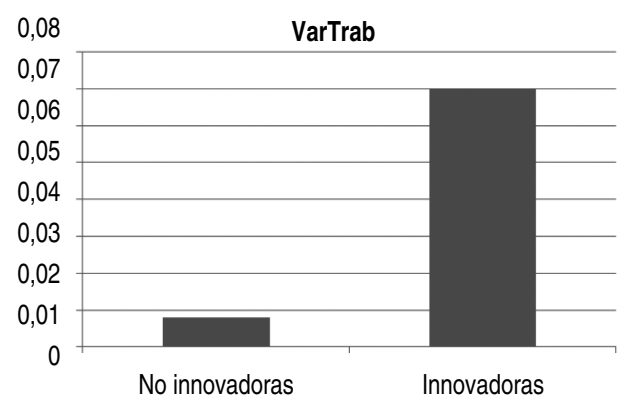

\section{Tabla 24. Prueba univariante. Plantilla}

\begin{tabular}{|lccc|}
\hline Ratio & Medias cuadradas & $\boldsymbol{F}$ & Significat. \\
\hline VarTrab & .0888 & 5.85 & 0.0157 \\
\hline
\end{tabular}

\section{7.- Conclusiones}

El actual contexto económico de globalización y de crisis exige que las cooperativas, al igual que las empresas capitalistas, mantengan y mejoren su competitividad, para lo cual deben plantearse nuevas estrategias de innovación en los productos y procesos de producción. La innovación debe considerarse fundamental para que exista un crecimiento a largo plazo con la consiguiente generación de empleo, razón por la cual los poderes públicos y los responsables de las empresas deben potenciarla.

En el estudio empírico que hemos realizado suponemos que la inversión en innovación beneficia a las empresas y por ende a la sociedad sobre todo por cuestiones laborales, razón por la que los organismos públicos suelen incentivar y financiar las inversiones necesarias en lo que se ha venido en lla$\operatorname{mar} I+D+i$.

En primer lugar, hemos clasificado las empresas -cooperativas en este caso- como innovadoras o no, siguiendo como criterio de pertenencia al grupo de las innovadoras el subordinado de la inver- 
sión en intangibles. Aunque los activos intangibles contabilizados no implican necesariamente más actividades innovadoras, existe una clara correlación entre estos dos conceptos. Por esta razón se ha tomado la inversión en estos activos como una aproximación o sinónimo de emprendimiento e innovación. Esta consideración limita en cierto modo los resultados obtenidos -posiblemente la utilización de otro criterio comporte la obtención de otros diferentes-. En trabajos como el de Goméz y Aleixandre (2014) sobre empresas de economía social de Castilla y León, se observa que las actividades de I + D están asociadas positivamente con la puesta en marcha de innovaciones de todo tipo (de producto, de proceso y organizativa).

Una vez agrupadas las sociedades cooperativas como pertenecientes a una u otra categoría (innovación y no innovación) y con la finalidad de identificar un mejor posicionamiento económico financiero de las mercantiles innovadoras en relación con las no innovadoras, hemos llevado a cabo diferentes análisis estadísticos tanto de índole univariante como multivariante.

En lo que a la rentabilidad se refiere, lo modelos estadísticos multivariantes no establecen diferencias entre las cooperativas innovadoras y las no innovadoras, lo que puede ser bastante lógico si tenemos en cuenta que la maximización de los beneficios económicos no suele ser el objetivo fundamental de este tipo de empresas. Sin embargo, las pruebas univariantes revelan que uno de los indicadores de rentabilidad es estadísticamente significativo y mayor para las innovadoras.

En cuanto al endeudamiento, en general es un poco mayor en las sociedades cooperativas innovadoras tal y como se desprende del análisis descriptivo, probablemente como consecuencia de las mayores inversiones que acometen este tipo de sociedades. El análisis de solvencia a largo plazo confirmaría estas apreciaciones pues uno de los ratios examinados ha resultado estadísticamente significativo, y dada su composición denotaría que las empresas innovadoras podrían tener unos mayores pasivos como consecuencia de un mayor endeudamiento para hacer frente a las actividades relacionadas con la innovación y los mayores plazos para recuperar este tipo de inversiones.

El análisis de solvencia a corto plazo y el de crecimiento nos ha permitido identificar que las mercantiles cooperativas innovadoras tienen un mayor volumen de negocio en comparación con las no innovadoras. Posiblemente el factor innovación en momentos de crisis como los actuales ayude a mantener las ventas o incluso a aumentarlas tal y como ha quedado claramente constatado tanto en los resultados univariantes como multivariantes del análisis del crecimiento.

Aunque los resultados de las pruebas univariantes y multivariantes indican que la fiscalidad no difiere entre los dos grupos de cooperativas analizadas, el estudio descriptivo revela que la media de la carga fiscal es menor para las cooperativas innovadoras, entre 2 y 5 puntos enteros dependiendo del ratio considerado. Detrás de esta diferencia se encontrarían las ventajas fiscales que obtienen las sociedades derivadas de sus inversiones en I+D+i. 
Por otro lado, del análisis de la variación de la plantilla se extrae que mientras en las sociedades no innovadoras la variación de la plantilla es prácticamente nula $(0,80 \%)$, en las innovadoras la media se ha incrementado en un 7,00\%, siendo esta diferencia estadísticamente significativa tal y como aparece reflejada en los resultados del análisis univariante. Teniendo en cuenta que para que una empresa pueda afrontar con garantías un proceso innovador, la formación y adaptación de los trabajadores resulta fundamental. Las cooperativas cuentan con la ventaja de disponer de un fondo de educación, formación y promoción que pueden emplear en parte para tal fin, facilitando la continuidad e incorporación de trabajadores en las empresas innovadoras, que serán a su vez las que tendrán mayores probabilidades de éxito empresarial. Esta relación positiva entre volumen de trabajadores e innovación queda también constatada en el trabajo de Gómez y Aleixandre (2014).

Siguiendo esta metodología, se pueden plantear estudios similares comparando diferentes sectores, comunidades autónomas y también con las empresas capitalistas tratando de establecer si hay diferencias importantes en lo que a las inversiones en actividades de innovación se refiere. También consideramos que podría ser muy interesante la realización de estudios parecidos de ámbito internacional, donde se incluyan empresas de países con una mayor tradición innovadora que el nuestro como Estado Unidos, Alemania, Reino Unido, Francia, Holanda, Dinamarca, Suecia, etc.

\section{8.- Bibliografía}

ÁGUILA OBRA, A.R. \& PADILLA MELÉNDEZ, A. (2010): "Factores determinantes de la innovación en empresas de economía social. La importancia de la formación y la actitud estratégica", CIRIEC España, Revista de Economía Pública, Social y Cooperativa, 67, 129-155.

ARUNDEL, A. (2007): "Innovation Survey Indicators: What Impact On Innovation Policy? Science, Technology, and Innovation Indicators in a Changing World: Responding to Policy Needs", OECD Blue Sky II Forum, Ottawa, Canada, Sept. 25-27, 2006. Published: September 2007, part 3, chapter 4.

BARRO, R.J. \& SALA I MARTÍN, X. (1995): Economic Growth, McGraw-Hill, Nueva York.

BASTERRETXEA, I. \& MARTÍNEZ, R. (2012): "Impact of management and innovation capabilities on performance: are cooperatives different?", Annals of Public and Cooperative Economics, 83, 3, 357-381.

BRETONES, F.D. (2004): "Actitudes, motivaciones y creación de empresas: el caso de los emprendedores en el sistema cooperativo", REVESCO, Revista de Estudios Cooperativos, 84, 37-52. 
CAPON, N., FARLEY, J.U. \& HOENIG, S. (1990): "Determinants of financial performance: a metaanalysis", Management Science, 36 (10), 1143-1159.

CARMONA, P., MOMPARLER, A. \& CLARA GIEURE, C. (2012): "The performance of entrepreneurial small and medium sized enterprises", The Service Industries Journal, 32 (15), 2463-2487.

COINVEST Proyect (www.coinvest.org.uk).

CEOE (2014): El estado de la innovación empresarial en España en 2014, informe elaborado por AVS Consulting.

CORRADO, C., HULTEN, C. \& SICHEL, D. (2006): "Intangible Capital and Economic Growth", Working Paper 11948. National Bureau of Economic Research, Cambridge, Massachusetts.

COTEC (2010): "La innovación en sentido amplio: un modelo empresarial. Análisis conceptual y empírico". Colección Innovación Práctica, COTEC, Madrid.

DOWLING, M. \& MCGEE, J. (1994): "Business and technology strategie and new venture performance. A study of the telecommunication equipment industry", Management Science, 12 (40), 1663-1677.

FERNÁNDEZ MORENO, M.V. \& PEÑA GARCÍA-PARDO, I. (2009): "Estrategia de innovación como factor determinante del éxito de la cooperativas vitivinícolas de Castilla-La Mancha", REVESCO, Revista de Estudios Cooperativos, 98, segundo cuatrimestre, 70-96.

GÓMEZ GARCÍA, J.M. \& ALEIXANDRE MENDIZÁBAL, G. (2014): "Economía social y comportamiento innovador: estudio empírico de las empresas de economía social en Castilla y Léon", CIRIECEspaña, Revista de Economía Pública, Social y Cooperativa, 81, 191-216.

GUIJARRO MARTINEZ, F. \& MOYA CLEMENTE, I. (2006): "Factores discriminantes de la inversión en intangibles de las empresas tecnológicas", Investigaciones Europeas de Dirección y Economía de la Empresa, 12 (2), 205-216.

GUZMÁN CUEVAS, J. \& MARTíNEZ ROMÁN, J.A. (2008): "Tipología de la innovación y perfiles empresariales: una aplicación empírica", Economía Industrial, 368, 59-77.

HILL, P. \& YOUNGMAN, R. (2003): "The Measurement of Intellectual Capital Formation in the System of National Accounts", PRISM WP5.

Ley 2/2011, de 4 de marzo, de economía sostenible.

Ley 14/2013, de 27 de septiembre, de apoyo a los emprendedores y su internacionalización.

Ley 27/2014, de 27 de noviembre, del Impuesto sobre Sociedades.

LEV, B. (2001): Intangibles: Management, Measurement and Reporting, Brookings Institution Press, Washington, D.C.

NAKAMURA, L. (2001): "What Is the U.S. Gross Investment in Intangibles? (At Least) One Trillion Dollars a Year!", Working Paper N ${ }^{\circ}$ 01-15. Federal Reserve Bank of Philadelphia, October. 
NELSON, R.R. (1996): The Sources of Economic Growth, Harvard University Press, Boston.

OCDE y Comunidad de Madrid (2007): Manual de Oslo. Directrices para la recogida e interpretación relativa a innovación, Sistema Madri+d.

PULIDO, A. (2009): "Una comparativa internacional en la medición del capital intangible de países", Revista de Economía Mundial, 23, 245-261.

Real Decreto 1514/2007, de 16 de noviembre, por el que se aprueba el plan General de Contabilidad.

Real Decreto-ley 3/2009, de 27 de marzo, de medidas urgentes en materia tributaria, financiera y concursal ante la evolución de la situación económica.

RODRÍGUEZ, J.M. \& GUZMÁN, C. (2013): "Innovation in social economy firms", Management Decision, 51, 5, 986-998.

ROSENBUSCH, N., BRINCKMANN, J. \& BAUSCH, A. (2011): "Is innovation always beneficial? A metaanalysis of the relationship between innovation and performance in SMEs", Journal of Business Venturing, 26 (4), 441-457.

SCHUMPETER, J.A. (1976): Teoría del Desenvolvimiento Económico, México: Fondo de Cultura Económica.

ZAHRA, S.A. \& BOGNER, W.C. (1999): "Tecnology strategy and software new venture's performance. Exploring the moderating effect of the competitive environment", Journal of Business Venturing, 15 (2), 135-173.

ZAHRA, S.A. \& COVIN, J. (1993): "Business strategy, technological policy and company performance", Strategic Management Journal, 14 (6), 451-478. 
